\title{
Near-Infrared Astrometry of Star Clusters with Different Flavors of Adaptive Optics and HST
}

\author{
Jessica R. Lu ${ }^{\mathrm{a}}$, Benoit Neichel ${ }^{\mathrm{b}}$, Jay Anderson ${ }^{\mathrm{c}}$, Evan Sinukoff ${ }^{\mathrm{a}}$, Matthew W. Hosek Jr. ${ }^{\mathrm{a}}$, \\ Andrea M. Ghez ${ }^{\mathrm{d}}$, Francois Rigaut ${ }^{\mathrm{e}}$, \\ anstitute for Astronomy, University of Hawaii, Honolulu, HI, USA; \\ bAix Marseille Université, CNRS, Laboratoire d'Astrophysique de Marseille, Marseille, France; \\ ${ }^{\mathrm{c}}$ Space Telescope Science Institute, Baltimore, MD, USA; \\ dUniversity of California, Los Angeles, CA, USA; \\ eRSAA, Australian National University, Mt Stromlo Observatory, ACT, Australia;
}

\begin{abstract}
High-precision infrared astrometry is a powerful tool for the study of resolved stellar populations throughout our Galaxy. We highlight two particular science cases that require precise infrared astrometry: (1) measuring the initial mass function in massive young clusters throughout the Milky Way and (2) finding isolated black holes that photometrically and astrometrically lens background bulge stars. Using astrometric results from these science cases, we perform a comparative analysis of the infrared astrometric capabilities from the Keck single-conjugate adaptive optics (AO) system, the Gemini multi-conjugate AO system, and the Hubble WFC3IR instrument. For the most crowded fields and a small region of interest, we show that Keck's single-conjugate AO system and the well-characterized NIRC2 instrument produce the highest astrometric precision at $~ 150 \mu$ as. However, for targets that cover a wider field of view, both the Gemini South AO Imager (GSAOI) and HST WFC3IR should be considered carefully. GSAOI currently delivers lower astrometric precision than HST WFC3IR for a given integration time; but, programs that require more frequent astrometric measurements over longer periods of time may benefit from the higher availability and possibly longer lifetime of GSAOI.
\end{abstract}

Keywords: adaptive optics, astrometry, star formation, star clusters, young stars

\section{INTRODUCTION}

High-precision relative astrometry has become a powerful tool for studying crowded stellar populations, particularly globular clusters, young massive clusters, and the Galactic center. At optical wavelengths, HST has demonstrated astrometric precisions of $<300$ microarcseconds on globular clusters. ${ }^{1}$ Relative proper motions have been used to measure clusters' internal dynamical state, search for intermediate mass black holes, and produce precise cluster memberships that reveal unexpected structures in color-magnitude diagrams. ${ }^{2-4}$ At infrared (IR) wavelengths, ground-based adaptive optics (AO) systems deliver the highest-resolution images and comparable or better astrometric precision, although over a smaller 10"-20" field of view (vs. 200" with the HST ACS instrument). AO astrometry at IR wavelengths has been critical for studies of stars orbiting the supermassive black hole at the Galactic center. ${ }^{5-9}$ Infrared astrometry is also necessary for studies of other heavily reddened populations. For instance, massive young clusters are ideal for determining whether the star-formation process and stellar initial mass function varies with environment as has been demonstrated on the Galactic center Arches and Quintuplet clusters ${ }^{10-12}$ and NGC 3603 and Westerlund 1 in the Milky Way disk. ${ }^{13,14}$ Studies of young massive clusters typically require larger fields of view than are delivered by most single-conjugate AO systems (e.g. Keck, VLT) and would benefit tremendously from high-precision astrometry from either multi-conjugate AO systems on the ground or high-resolution infrared imagers in space. Another example science case is the astrometric study of individual long-duration microlensing events towards the Galactic bulge, which can reveal whether the lensing object is an isolated stellar-mass black hole. The lens produces both a photometric magnification and an astrometric deviation as a background star moves behind the lens and these measurements can be

Further author information: (Send correspondence to J. R. Lu)

J. R. Lu: E-mail: jlu@ifa.hawaii.edu, Telephone: 1808 956-8573

Adaptive Optics Systems IV, edited py Enrico Marchetti, Laird M. Close,

Jean-Pierre Véran, Proc. of SPIE Vol. 9148, 91480B · (c) 2014 SPIE

CCC code: $0277-786 \mathrm{X} / 14 / \$ 18 \cdot$ doi: $10.1117 / 12.2057241$

Proc. of SPIE Vol. 9148 91480B-1 
combined to directly measure the mass of the lens. ${ }^{15-20}$ This science case needs maximum astrometric precision over a small field of view, which is well matched to single-conjugate AO systems.

In this proceeding, we present preliminary astrometric results from these two science cases as a means of quantifying and comparing the astrometric capabilities of the single-conjugate AO Keck NIRC2 instrument, the multi-conjugate AO Gemini GSAOI instrument, and the HST WFC3IR instrument. In $\S 2$, we present the current astrometric precision and accuracy from ground-based $\mathrm{AO}$ systems. In $\S 3$, we present astrometric results from the HST WFC3IR instrument and a comparative analysis with AO astrometry is presented in $\S 4$. Finally, in $\S 5$ we present preliminary results derived from astrometry on both the study of young, massive clusters and the search for microlensing stellar mass black holes.

\section{IR ASTROMETRY WITH ADAPTIVE OPTICS}

\subsection{Keck Single Conjugate Adaptive Optics}

The facility near infrared camera on Keck II (NIRC2, PI: K. Matthews) at the W. M. Keck Observatory is mounted on a stable Nasmyth platform behind a single-conjugate laser-guide star adaptive optics (AO) system. ${ }^{21}$ This camera and AO system has become a workhorse for high-precision astrometric observations thanks to its optical stability and significant efforts to develop astrometric reduction techniques ${ }^{22,23}$ and characterize the geometric distortion. ${ }^{24}$ Much of the characterization was undertaken as part of the UCLA Galactic Center program in which precise astrometry is used to measure the orbits of stars around our Galaxy's supermassive black hole. ${ }^{6}$ Currently, relative astrometric precisions of $80 \mu$ as and accuracies of $150 \mu$ as are routinely delivered over a 10" $\times 10$ " field of view on the Galactic Center,${ }^{9}$ which is typically observed astrometrically under median or better seeing conditions. A similar floor of $150 \mu$ as in astrometric accuracy is also seen on the NACO system at the VLT and the source of this systematic astrometric error is likely a combination of errors in the point spread function (PSF), which is estimated from the data and is assumed to be constant over the field, and residual distortions. ${ }^{25}$ Efforts are underway to improve both the on-axis PSF estimation ${ }^{26,27}$ and the off-axis PSF spatial variability (UCLA AO Optimization project, PI: Ghez) to reduce this astrometric floor.

In addition to Galactic center observations, a number of new science cases are possible with relative astrometric errors of $150 \mu$ as. For instance, we have conducted a pilot project to detect isolated black holes in the Milky Way that microlens background bulge stars (see 5.2 for more details). We highlight this project, as it represents what can reasonably be expected on stellar populations that are both fainter and less crowded than the Galactic center. This project required astrometric measurements obtained roughly once a month during the photometric peak of the lensing event and then less frequent follow-up measurements over several years. Given the necessary time sampling and classical telescope scheduling, there was no allowance for poor weather; therefor, the resulting quality of the astrometric measurements more broadly sample the range of seeing conditions that a typical non-queue observing program would experience and are not as precise as the Galactic center case.

NIRC2 astrometric observations were obtained for the OGLE-selected, long-duration microlensing event, OB110022 from 2011-2013 (Sinukoff et al., in preparation). The observations were designed to minimize relative astrometric errors down to $\mathrm{K}=18$, which is $2-3$ magnitudes fainter than past observations. Integration times were set such that the brightest star in the field, which in this case is the lens at $\mathrm{K}=13$, yields counts in the peak pixel that are just short of the regime where detector non-linear flux response becomes an issue (12,000 DN for NIRC2). The integration time per exposure is tuned for each run depending on the seeing and cloud-cover and was typically $60 \mathrm{~s}$. These integration times are longer than in Galactic Center observations and benefit from reduced readnoise due to large numbers of Fowler samples when reading the detector. In each epoch, 30-50 exposures were taken and only small $\left(<0^{\prime \prime} .7\right)$ dithers were used to remove bad pixels while minimally sampling optical distortions. The resulting astrometric precision after combing all exposures in a single epoch was 240 $\mu$ as at $\mathrm{K}=18$, with some epochs as high as $340 \mu$ as in poor ( $>1$ ") seeing and some epochs as low as $110 \mu$ as in good $\left(\sim 0^{\prime \prime} 6\right)$ seeing (Figure 1, left). To derive the relative astrometric accuracy, we compare the measured proper-motion uncertainties with those predicted from

$$
\sigma_{\mu}=\frac{\sigma_{\mathrm{pos}}}{\left[\sum_{i}\left(t_{i}-t_{o}\right)^{2}\right]^{1 / 2}}
$$


where $\sigma_{\text {pos }}$ is the typical astrometric precision per epoch and $t_{i}$ is the date of each epoch. In the case of OB110022, the proper-motion uncertainties are $65 \%$ higher that expected (200 $\mu$ as/yr vs. $120 \mu$ as/yr). The source of this discrepancy is likely the severe anisoplanatism and PSF variability present in several of the epochs, which we dealt with by restricting our analysis to a circular field of view with a radius of 2.5 at the expense of losing reference stars.
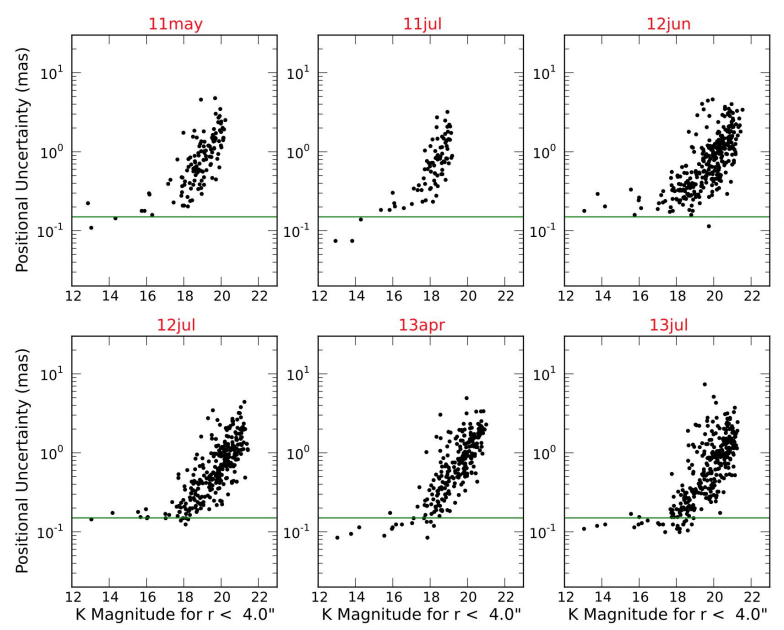

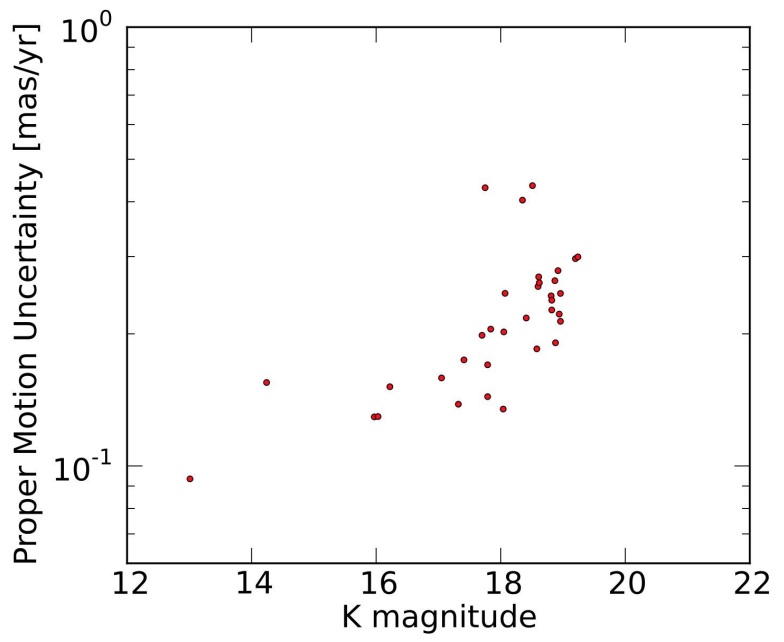

Figure 1. Keck single-conjugate adaptive optics positional uncertainties (left) and proper-motion uncertainties (right) for six epochs spread over a two years on the candidate black hole lens, OB110022. The observations were designed to reach a systematic astrometric floor of $150 \mu$ as at $\mathrm{K}=18$ in each epoch (green line), which takes 30-50 minutes of integration time. The resulting proper-motion errors are slightly larger than that predicted by Equation 1, most likely due to ignoring PSF spatial variability and errors in cross-epoch coordination transformations. Proper motions errors are shown for the subset of stars that are detected in all 6 epochs and are within $2^{\prime \prime} .5$ of the target source.

\subsection{Gemini Multi Conjugate Adaptive Optics}

The Gemini Multi Conjugate Adaptive Optics System, GEMS, ${ }^{28,29}$ feeds the GSAOI instrument, ${ }^{30,31}$ both of which are mounted at a Cassegrain port behind the primary mirror on the Gemini South Telescope. Gemini GSAOI has an $85 " \times 85$ " field of view, substantially larger than the single-conjugate AO system that feeds Keck NIRC2. During commissioning, the cluster NGC 1851 was observed over several nights (Figure 2) and the data have been analyzed to characterize the astrometric performance of the system. Figure 3 shows the resulting astrometric precision as a function of stellar brightness from 10 minutes of data obtained 2013 March 04. The same reduction tools were utilized to analyze this data as were developed for Keck NIRC2 data. ${ }^{22}$ Unlike the Keck NIRC2 data, the Gemini data showed substantial improvements in the astrometric precision when a high 2nd-order polynomial was used to transform the coordinates of each image into a common reference frame (Figure 4).

While the astrometric precision within a single observation appears to be comparable to Keck NIRC2, only a multi-epoch analysis can be used to test the astrometric accuracy of the system. Fortunately, NGC 1851 was observed on two consecutive nights for $\sim 170$ seconds each night with no dithers, the same natural guide stars, and stars located on the same position of the detector - a nearly ideal setup for testing some components of astrometric accuracy. A comparison of the stellar positions over the two nights shows that the astrometry is less accurate than predicted based on the single-night precision (Figure 5). This is likely the result of time-variable distortion induced by gravity vector changes as the telescope points at different elevations. ${ }^{28}$ The elevation angle differed by $10^{\circ}$ across the two nights. The high-order transformation removes most of the time-variable distortion. However, some spatial correlations remain, as evidenced by the asymmetry in the right panel, suggesting that even higher-order residual distortions are still present. ${ }^{29}$ 


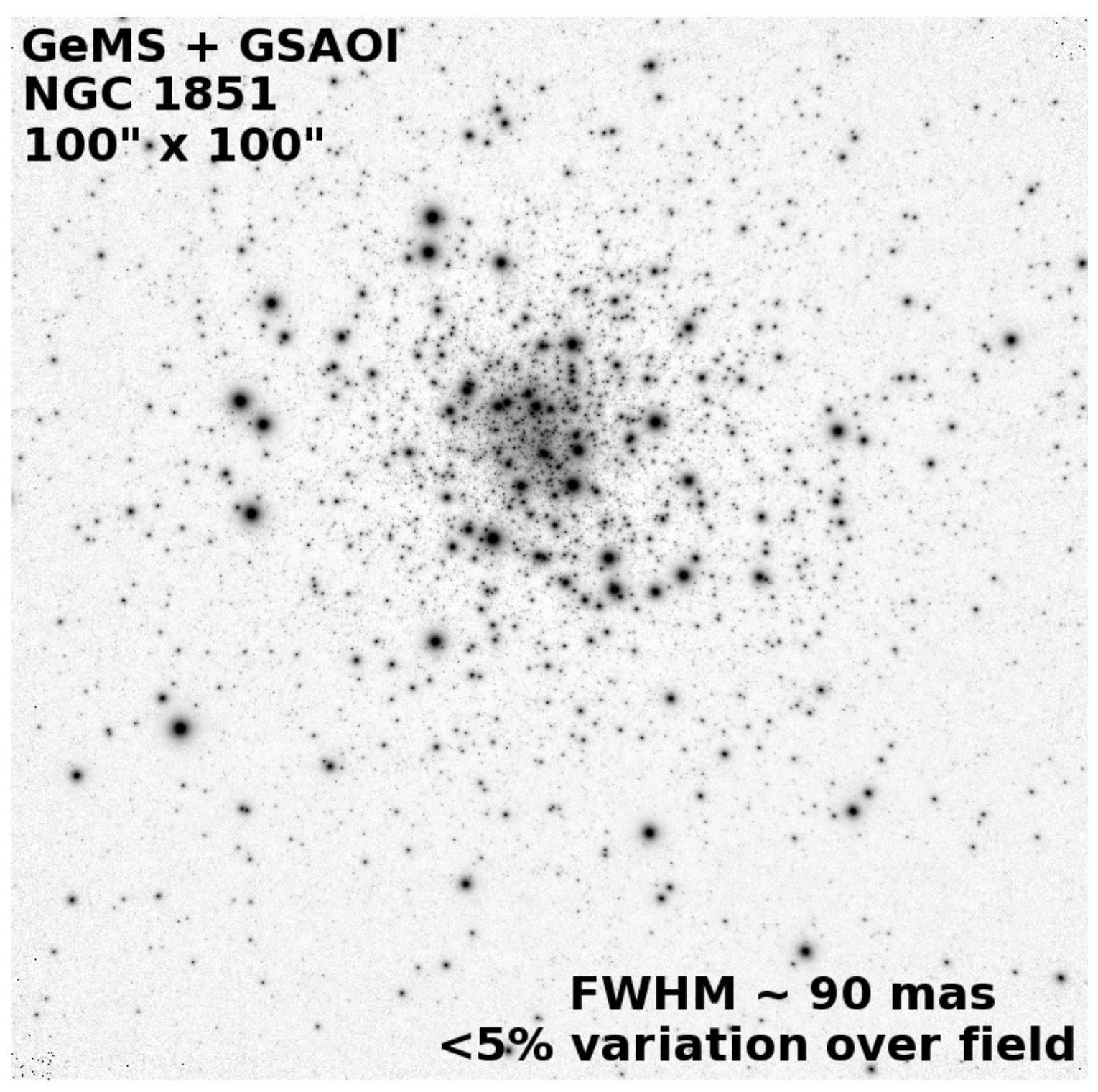

Figure 2. A GSAOI K-band mosaic image of the cluster NGC 1851 taken during GeMS commissioning. ${ }^{28,29}$ This data $^{2}$ set demonstrates the uniform and high-resolution image quality over a 85 " $\times 85$ " field of view (mosaiced to 100 " $\times 100$ ") that the system delivers. The spatial resolution is a factor $\sim 1.5$ better than the resolution delivered by HST at nearinfrared wavelengths, although HST's higher Strehls yield better contrast. The relatively uniform PSF that GeMS delivers is essential for high-precision astrometry since a spatially varying PSF is likely the source of the current systematic astrometric floor of Keck single-conjugate AO data.

\section{IR ASTROMETRY WITH HST WFC3}

The Wide Field Camera 3 (WFC3) on the Hubble Space Telescope (HST) offers infrared imaging over a larger field of view than any current ground-based AO system at the expense of lower spatial resolution due to HST's smaller aperture and poorer pixel sampling. To compare, WFC3IR, GSAOI, and NIRC2 have fields of view of 130", 85", and 10" typical spatial resolutions of 156 mas at H-band, 90 mas at H- or K-band, and 60 mas at K-band, respectively. HST also has the advantage of residing above the Earth's atmosphere and does not suffer from the residual atmospheric jitter that dominates astrometry with ground-based AO at short integration times. Although ground-based AO systems show more PSF variations on short timescales, HST's PSF does 


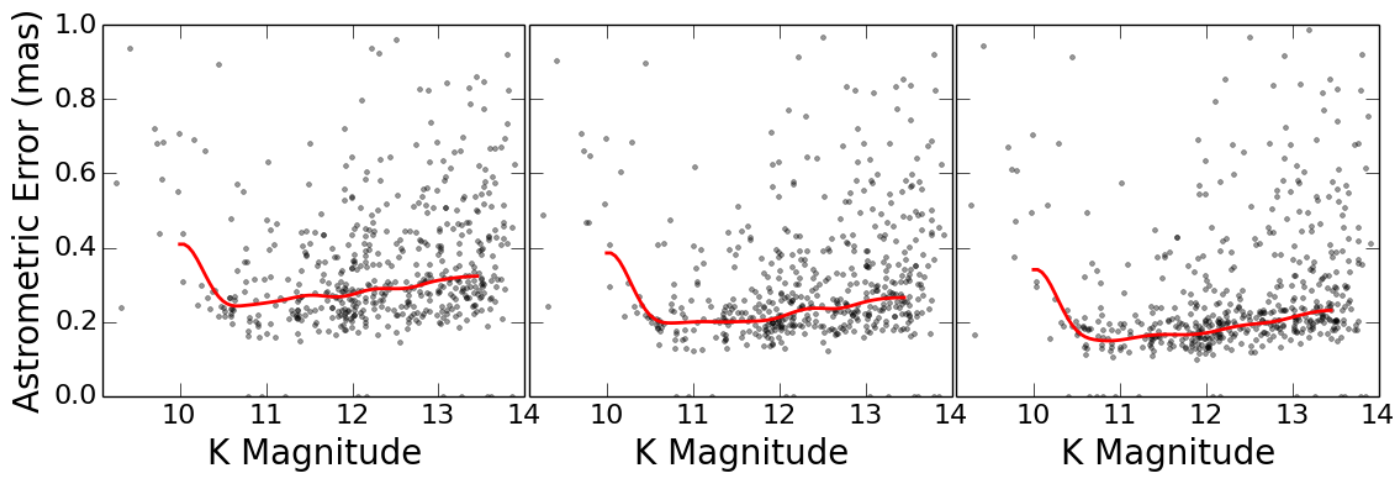

Figure 3. GeMS+GSAOI positional uncertainties vs. magnitude with individual frames transformed into a common coordinate system with polynomials of increasing order: Left: Translation, rotation, and scale (4 free parameters). Middle: Full first-order polynomial (6 free parameters). Right': Full second-order polynomial (12 free parameters). The median positional error at different magnitudes (black line) shows the effects of saturation for stars brighter than $\mathrm{K}<10.5$ and only slight increases down to $\mathrm{K}=14$. The median positional uncertainties below $\mathrm{K}>12$ show substantial improvement with the higher order transformations going from $300 \mu$ as (left) to $200 \mu$ as (right). These commissioning observations were limited to a total integration time of only 10 minutes and show comparable astrometric precision to the Keck single-conjugate AO data when scaled by $t_{i n t}^{-1 / 2}$.
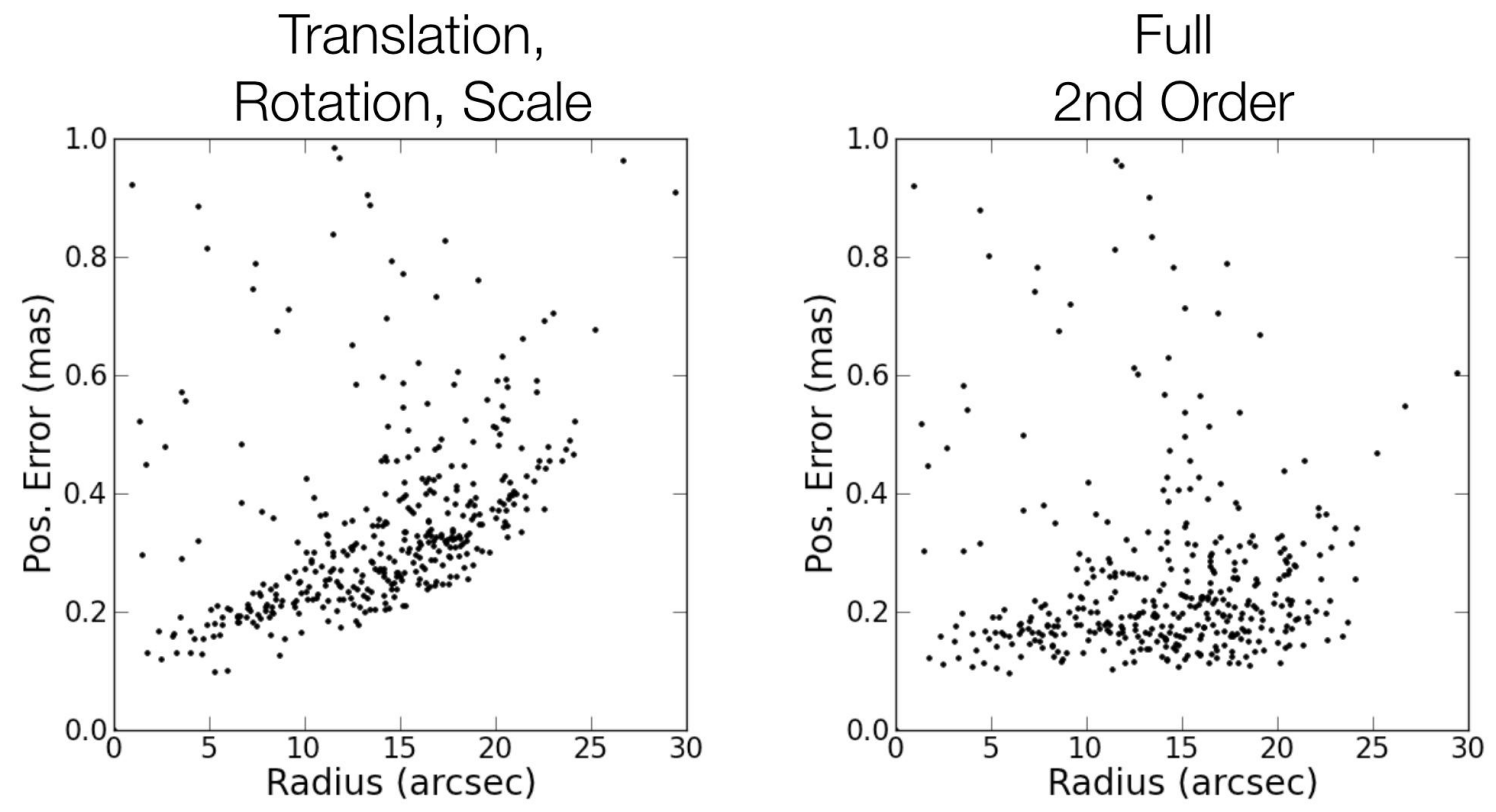

Figure 4. GeMS+GSAOI positional uncertainties vs. radius with individual frames transformed into a common coordinate system with polynomials of increasing order: Left: Translation, rotation, and scale (4 free parameters). Right': Full secondorder polynomial (12 free parameters). The data were undithered and should not be affected by static optical distortions. The dramatic improvement from higher-order polynomial transformations suggests that there is a time-variable component to the distortion. The likely source of the time-variable distortion is gravity-induced flexure as these observations were spread over 1 hour with an elevation change of $8^{\circ}\left(66^{\circ}-75^{\circ}\right)$. 

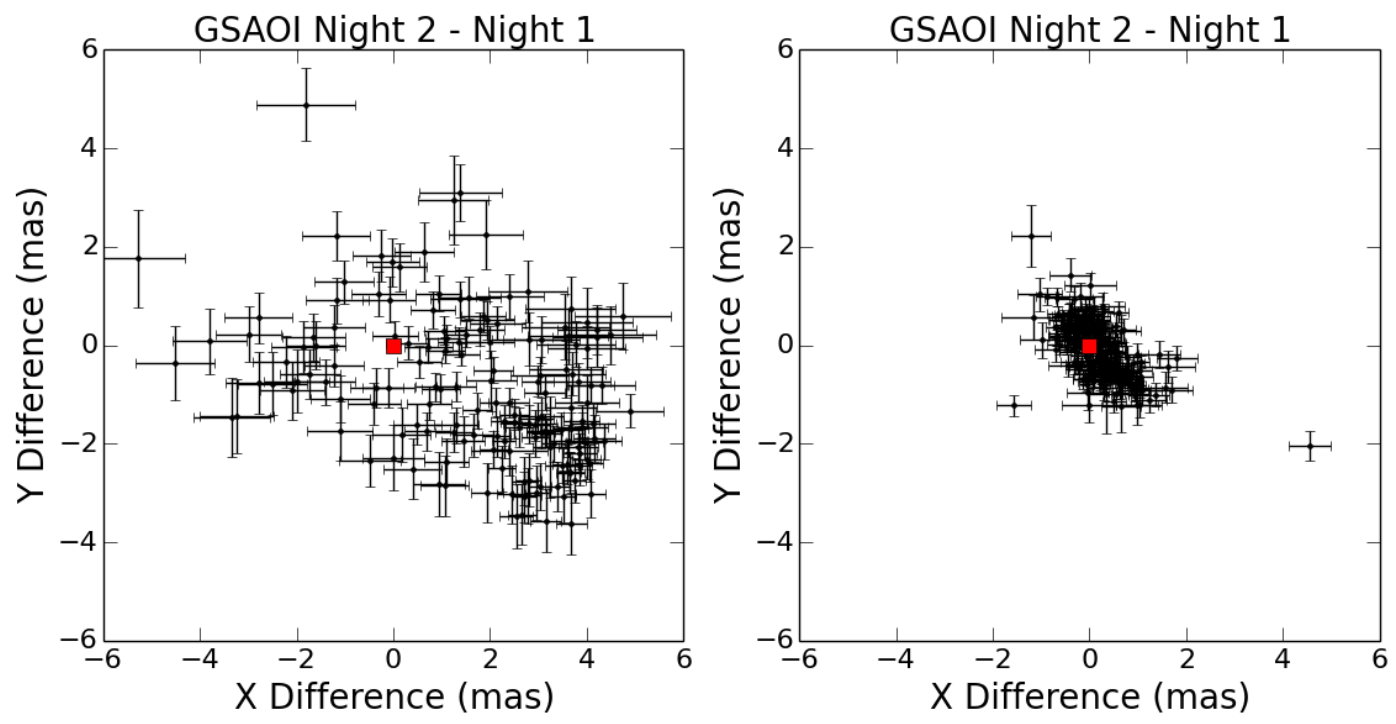

Figure 5. Difference between star positions measured on two consecutive nights with individual frames transformed into a common coordinate system with polynomials of increasing order: Left: Translation, rotation, and scale (4 free parameters). Right': Full second-order polynomial (12 free parameters). The RMS of the positional differences is $\sim 2000 \mu$ as in the low-order case and $600 \mu$ as in the high-order case. The error bars show the combined normal errors from each night, 790 $\mu$ as and $460 \mu$ as for the low- and high-order transformation, which are substantially smaller than the observed scatter. This is likely the result of time-variable distortion induced by gravity vector changes as the telescope points at different elevations. The elevation angle differed by $10^{\circ}$ across the two nights. The high-order transformation removes most of the time-variable distortion. However, some spatial correlations remain, as evidenced by the asymmetry in the right panel, suggesting that even higher-order residual distortions are still present.

change appreciably between exposures due to slow thermal variations in the satellite. Fortunately, the timevariable component of the PSF can be treated as a small perturbation on the time-averaged PSF and the perturbation is nearly uniform over the field of view. This is offers a distinct advantage over ground-based AO where both the on-axis PSF and the PSF spatial variations must be determined separately for each image.

Given the astrometric success of HST's optical cameras, we have embarked on a study of several massive $\left(10^{4}\right.$ $\left.\mathrm{M}_{\odot}\right)$, young $(<10 \mathrm{Myr})$ clusters (Westerlund 1, Arches, Quintuplet) to determine if the initial mass function differs from that found in the local solar neighborhood or at the Galactic Center (see 5.1 for more detail). We use this program to illustrate the astrometric capabilities of WFC3IR in comparison with ground-based AO astrometry. The HST observations have been carefully designed with dithers landing at different pixel phases in order to compensate for the under-sampled pixels (1.2 pixels per resolution element) and only small dithers $(<10$ ") were used to avoid introducing optical-distortion errors. Observations were obtained once a year for three years on the Arches cluster with stars placed at identical positions on the camera in all epochs (Hosek et al., in preparation). The total integration time was $\sim 200$ minutes per epoch. One difficultly with HST WFC3IR observations is the slow detector readout times and small on-instrument buffer, which makes it exceedingly difficult to obtain the short-exposures that are necessary to measure bright stars without saturation. Drift scan techniques applied in other bright-star astrometry cases are not applicable due to high stellar densities in our clusters. The most heavily saturated stars cannot be accurately subtracted and should be masked out in both the core and the extended diffraction spikes during astrometric analysis.

Precise astrometry is extracted from the images using tools initially developed for HST's WFPC2 and ACS instruments. ${ }^{1}$ Key factors for WFC3IR astrometry include modeling the PSF spatial variability using a $3 \times 3$ grid of PSFs and also allowing the PSFs to change by small, smooth perturbations from exposure to exposure. Astrometric errors are at least a factor of 2 higher if a uniform, static PSF is used instead. We also note that archival observations that are not designed for astrometry (with insufficient pixel phase sampling or widely 

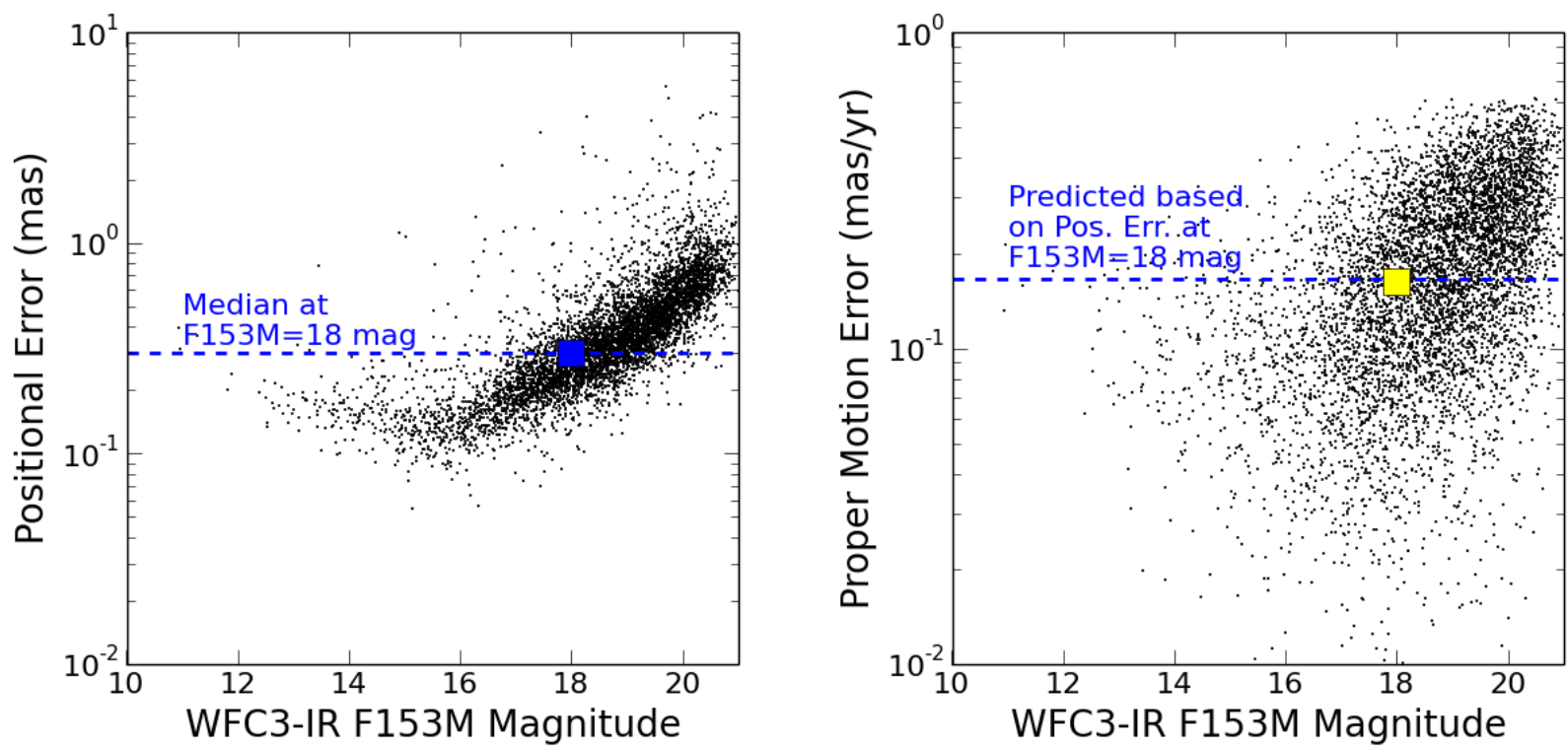

Figure 6. HST WFC3IR positional uncertainties (left) and proper-motion uncertainties (right) on the Arches star cluster in the Galactic center in the F153M ( $\sim$ H-band) filter (Hosek et al., in preparation). The median positional uncertainty at $\mathrm{F} 153 \mathrm{M}=18 \mathrm{mag}$ is $300 \mu \mathrm{as}$ and should yield a predicted proper-motion error, based on Equation 1 , of $170 \mu \mathrm{as} / \mathrm{yr}($ blue dashed line). The resulting median proper-motion uncertainty at F153M=18 mag is $0.160 \mu$ as/yr, in agreement with the prediction, showing that there are no uncaptured sources of systematic error in the relative astrometry at these faint magnitudes. Saturation and non-linearity introduces additional error for stars brighter than F153M<16. Despite HST's lower spatial resolution, the astrometric precisions of these observations are comparable to the higher-resolution AO observations shown in Figure 1 thanks to longer integration times, higher SNR, and a precise estimate of the PSF and its spatial variability for the HST data.

dithered) may yield astrometric errors between 2 and 7 mas due to sub-Nyquist centroiding errors and residual distortions. For the carefully designed Arches experiment (Hosek et al, in preparation), the resulting astrometric precision per epoch has a minimum of $170 \mu$ as at F153M-band=16 and grows to $300 \mu$ as at F153M=18 (Figure 6 , left). Note that the astrometric uncertainties are taken as the error on the mean position rather than the standard deviation of the positions measured in each image, which is consistent with the error analysis of the AO observations. We find that predicted proper-motion uncertainties based on Equation 1 match with the observed proper-motion uncertainties for faint stars (Figure 6). However, the bright stars astrometric errors are slightly larger than expected suggesting that there is a small additional source of systematic error.

\section{COMPARISON OF AO VS. HST INFRARED ASTROMETRY}

Currently, the astrometric analysis of AO and HST data are at different stages of development. HST has nearly a decade head-start and is a more stable system when compared with ground-based AO systems. Nevertheless, we present a brief comparison of the strengths and weaknesses of each system in an effort to help guide users interested in performing new astrometric experiments. In order to more directly compare WFC3IR, Gemini GSAOI, and Keck NIRC2, we must estimate the single-epoch astrometric accuracy in an integration time of 15 minutes at $\mathrm{K}=18 \mathrm{mag}$. The random component of the positional uncertainty is assumed to scale with integration time and flux as $t_{\text {int }}^{-1 / 2}$ and $f^{-1 / 2}$. For Keck NIRC2, we adopt a $240 \mu$ as astrometric accuracy at $\mathrm{K}=18$ in 30 minutes of integration time composed of $190 \mu$ as of random error and a $150 \mu$ as systematic contribution. This scales to $370 \mu$ as at $\mathrm{K}=18$ in 15 minutes. For Gemini GSAOI, we assume the stellar density of the observations is sufficiently high that high-order transformations can be used between all images. The resulting astrometric accuracy is $600 \mu$ as at $\mathrm{K}=14$ in 3 minutes and is composed of $460 \mu$ as of random error and $385 \mu$ as of systematic 

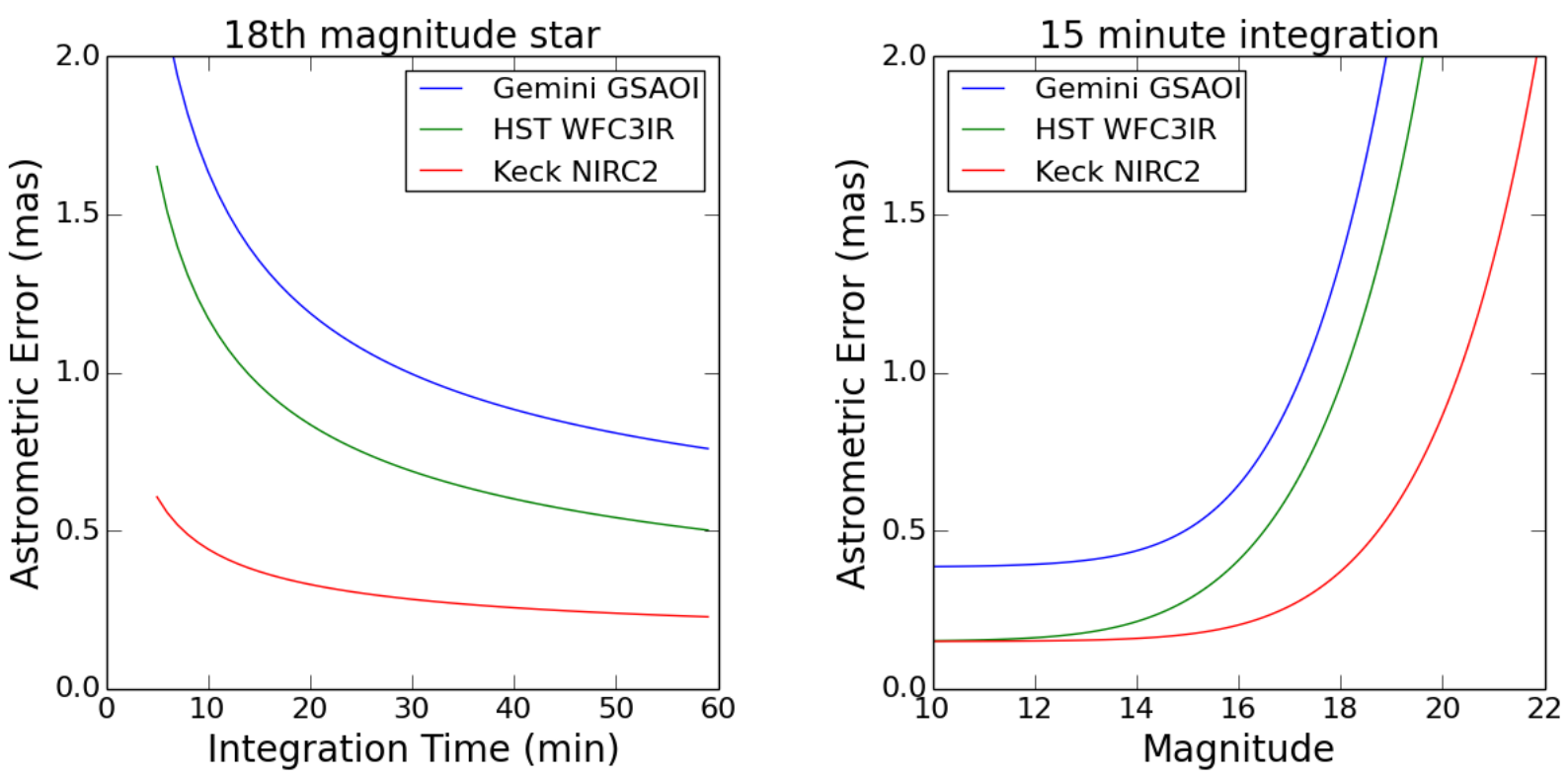

Figure 7. Comparison of astrometry with Keck NIRC2, Gemini GSAOI, and HST WFC3IR as a function of integration time (left) and stellar magnitude (right). See $\S 4$ for details. All astrometric errors are assumed to scale with integration time and flux as $t_{i n t}^{-1 / 2}$ and $f^{-1 / 2}$ and to reach a systematic astrometric floor that is unique to the analysis of each instrument. The systematic floor has not been full explored for Gemini GSAOI and HST WFC3IR and (1) may not exist, (2) may scale with the properties of the observations, and (3) may be the result of different processes than for Keck NIRC2.

error, which may be appropriate for observations taken with a $10^{\circ}$ elevation spread. This scales to $1350 \mu$ as at $\mathrm{K}=18$ in 15 minutes. For HST WFC3IR, we adopt an error of $300 \mu$ as at $\mathrm{K}=18$, assuming the same sensitivity between F153M-band and K-band, in 200 minutes, which is composed of $260 \mu$ as of random error and $150 \mu$ as of systematic error. We note that the systematic floor has not been verified on HST WFC3IR with different lengths of observations; however, the astrometric error appears too large at F153M=16 as compared with only flux-scaled predictions at $\mathrm{F} 153 \mathrm{M}=18$ and a systematic floor resolves the issue. This scales to $960 \mu$ as at $\mathrm{K}=18$ in 15 minutes. A summary of the scaled values is presented in Table 1 and Figure 7 shows estimated astrometric errors for different integration times and magnitudes.

Table 1. Comparison of astrometric errors across instruments for an 18th magnitude star in 15 minutes.

\begin{tabular}{lr}
\hline $\begin{array}{l}\text { Instrument } \\
()\end{array}$ & $\begin{array}{r}\text { Astrometric Error } \\
(\mu \text { as })\end{array}$ \\
\hline Keck NIRC2 & 370 \\
Gemini GSAOI & 1350 \\
HST WFC3IR & 960 \\
\hline
\end{tabular}

For the most crowded fields and a small region of interest, it is clear that Keck's single-conjugate AO system and the well-characterized NIRC2 instrument are superior. However, for targets that cover a wider field of view, the decision-tree is more complex. Gemini GSAOI and HST WFC3IR have similarly large fields of view and Gemini is queue scheduled such that good image quality can be required. Gemini GSAOI has better spatial resolution, but larger astrometric errors than HST WFC3IR. The most crowded wide-field science cases will require the larger aperture of Gemini GSAOI. The highest-precision, wide-field science cases where over-crowding is less of a concern will benefit from the better precision on HST WFC3IR. However, the HST subscription rate 
is higher than Gemini South by a factor of $4-5$, while the astrometric errors differ by a factor of 1.5 . A detailed comparison of the overheads between HST and Gemini for a specific program is necessary to determine which instrument is more suitable. One cautionary note, Gemini GSAOI is the newest facility and its astrometric properties (e.g. distortion solution) are still under development.

\section{SCIENCE APPLICATIONS}

There are strong science motivations for developing high-precision infrared astrometry capabilities. In this section, we highlight two cases: (1) measuring the initial mass function in massive young clusters and (2) finding isolated black holes that microlens background bulge stars.

\subsection{Young Massive Clusters}

Massive star clusters are ideal laboratories for studying a wide range of stellar phenomena and are the closest observable realization of the simple stellar population models that are used throughout astronomy. The stars in these clusters most likely formed at the same time and with the same chemical composition; only the mass of each star differs (although see ${ }^{32,33}$ ). For this reason, massive globular clusters have become the de facto test bed for stellar evolution and stellar atmosphere models. ${ }^{34-36}$ The youngest and most massive clusters $\left(>10^{4} \mathrm{M}_{\odot}\right)$ are particularly worthy targets as they contain many stars even at the highest masses $\left(150 \mathrm{M}_{\odot}\right)$ and allow us to probe the earliest phases of stellar and cluster evolution and measure the initial mass function. However, these young massive clusters are typically found deep in the Galactic plane at large distances and high extinction. Confusion between cluster members and foreground and background field stars often limits the detailed study of these clusters. Proper motions largely resolve this issue since cluster members move together and can be separated from the contaminating field stars. ${ }^{10-14}$ Figure 8 shows the proper motions of stars in and around the Westerlund 1 (Wd 1) young massive cluster derived from archival optical and near-infrared HST images. Members are selected based on their common proper motions and reveal the cluster sequence from an otherwise complex CMD. The cluster CMD shows a nearly vertical main sequence, as expected in the infrared, and the pre-main-sequence turn-on point around $2 \mathrm{M}_{\odot}$. The proper motions are currently derived with optical images as the first epoch and are thus limited to $\sim 1 \mathrm{M}_{\odot}$. Repeated IR observations have been obtained and will allow us to identify members and reconstruct the initial mass function of the cluster down to $\sim 0.2 \mathrm{M}_{\odot}(\mathrm{Lu}$ et al., in preparation). We have embarked on similar programs with Keck NIRC2 and Gemini GSAOI for several other massive young clusters at different ages and locations throughout the Milky Way.

\subsection{Black Holes Microlensing Background Bulge Stars}

The only black hole remnants of massive stars that have been definitively detected are $\sim 30$ that are accreting from companions. ${ }^{37}$ However, there may be over 100 million isolated stellar mass black holes within the Milky Way. ${ }^{38}$ One method for detecting isolated black holes is through a microlensing event in which the black hole lenses a background star as they pass within close projected (not three-dimensional) proximity. ${ }^{15-20}$ Black holes with masses $>5 \mathrm{M}_{\odot}$ should produce microlensing events with long-durations (>100 days) and high magnification that can easily be identified using OGLE or MOA surveys of the Galactic bulge. However, the long-durations can also be produced with a less massive lense if the lensing object and the background source have a similar proper motion on the sky. Fortunately, microlensing produces an astrometric deviation that is proportional to the mass of the lens, which can be used to determine if the lens is truly a black hole. In 2011, we embarked on a pilot project to identify candidate black hole microlenses that showed evidence for long-durations as their flux increased to the photometric peak. We obtained multi-epoch Keck NIRC2 astrometry immediately after the peak and for several years after in order to search for non-linear astrometric motions. No significant non-linear motions have been detected yet, largely due to very poor atmospheric conditions in 2011. However, we have demonstrated the feasibility of the technique and two of the objects in our sample have sufficiently long durations that they need to be re-observed over the next two years to estimate the proper motion of the background source after it is no longer lensed. Figure 9 shows the expected astrometric deviation from linear proper motion for a $10 \mathrm{M}_{\odot}$ black hole, which may be measurable by 2016 for OB110022 (Sinukoff, in preparation). 

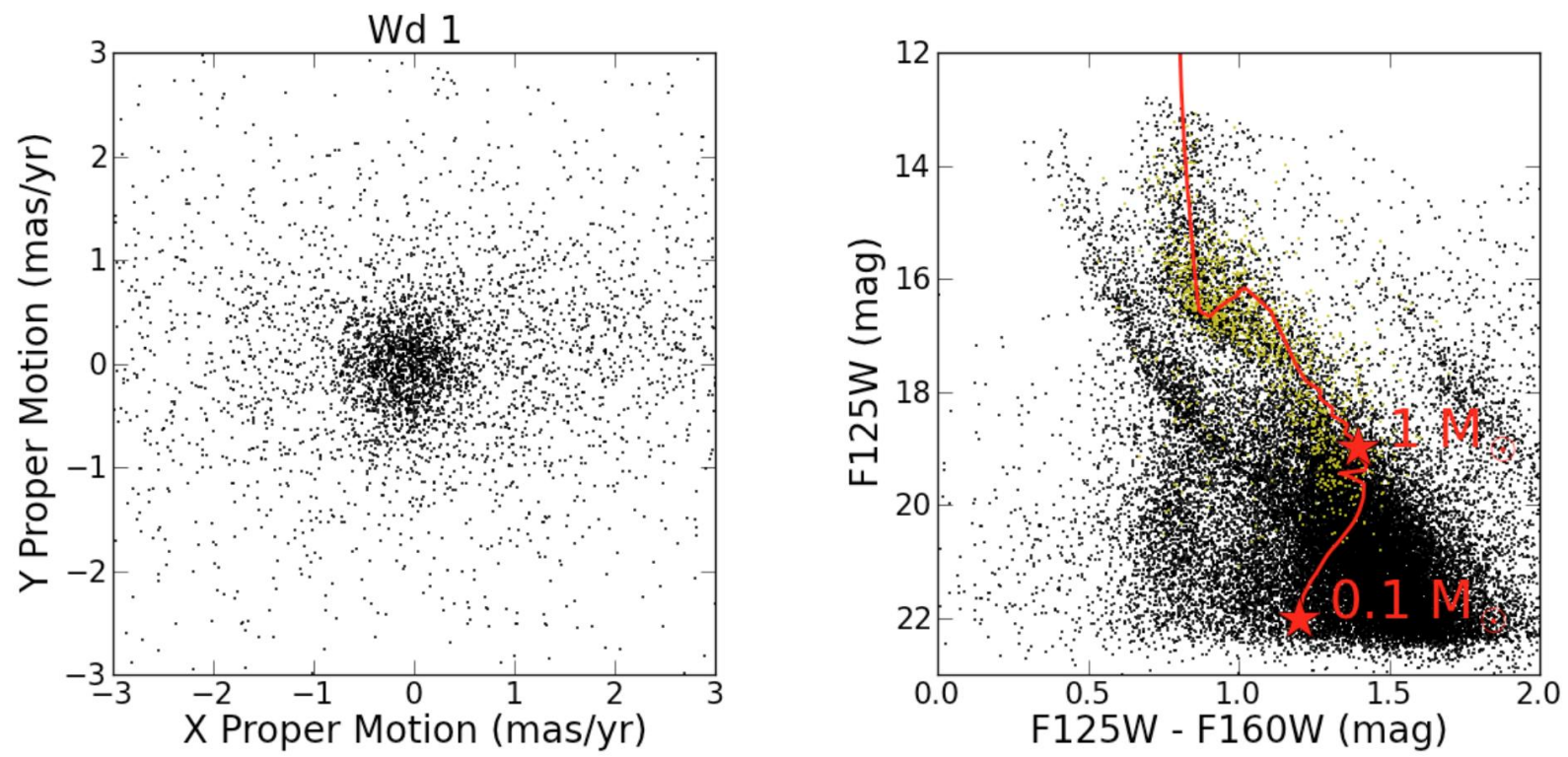

Figure 8. Proper motions (left) and color-magnitude diagram (CMD, right) for the massive young cluster, Wd 1. Highprecision astrometry allows cluster members to be separated from contaminating field stars since cluster members share a common proper motion. Cluster members selected in this manner (right, yellow points) reveal the cluster sequence from an otherwise complex CMD. The cluster CMD shows a nearly vertical main sequence, as expected in the IR, and the pre-main-sequence turn-on point around $2 \mathrm{M}_{\odot}$. These HST proper motions were extracted from archival optical and IR images and are thus limited to $\sim 1 \mathrm{M}_{\odot}$. Second-epoch IR observations have been obtained and will allow us to identify members and reconstruct the initial mass function of the cluster down to $\sim 0.2 \mathrm{M}_{\odot}$.

\section{CONCLUSIONS}

We have presented a comparative astrometric analysis of the single-conjugate (Keck NIRC2) and multi-conjugate adaptive optics (Gemini GSAOI) systems and HST WFC3IR. We have highlighted several science cases that benefit from high-precision infrared astrometry over both small (10") and large (90") fields of view and showed preliminary astrometric results. The benefits of high-precision infrared astrometry both from ground-based AO systems and from HST have not diminished with the launch of Gaia. Gaia will have high astrometric errors in the crowded-stellar-population science cases presented here. Furthermore, Gaia's optical sensitivity limit is $\mathrm{V}<20$, which corresponds to stellar masses above $15 \mathrm{M}_{\odot}$ for Westerlund 1 , one of the least reddened young, massive clusters. The Galactic center clusters, such as the Arches and Quintuplet, are undetectable with Gaia. Development of AO astrometric analysis techniques and system characterization is still at the early stages, particularly for the new Gemini GSAOI system, and is critical to the design of future AO systems.

\section{ACKNOWLEDGMENTS}

J. R. Lu acknowledges support from the NSF Astronomy and Astrophysics Postdoctoral Fellow program (AST-1102791). B. Neichel acknowledges support from the French ANR program WASABI-ANR-13-PDOC-0006-01. Some of the data presented herein were obtained at the W.M. Keck Observatory, which is operated as a scientific partnership among the California Institute of Technology, the University of California and the National Aeronautics and Space Administration. The Observatory was made possible by the generous financial support of the W.M. Keck Foundation. The authors wish to recognize and acknowledge the very significant cultural role and reverence that the summit of Mauna Kea has always had within the indigenous Hawaiian community. We are most fortunate to have the opportunity to conduct observations from this mountain. Some of the results were based on observations obtained at the Gemini Observatory, which is operated by the Association of Universities for Research in Astronomy, Inc., under a cooperative agreement with the NSF on behalf of the Gemini partnership: the 


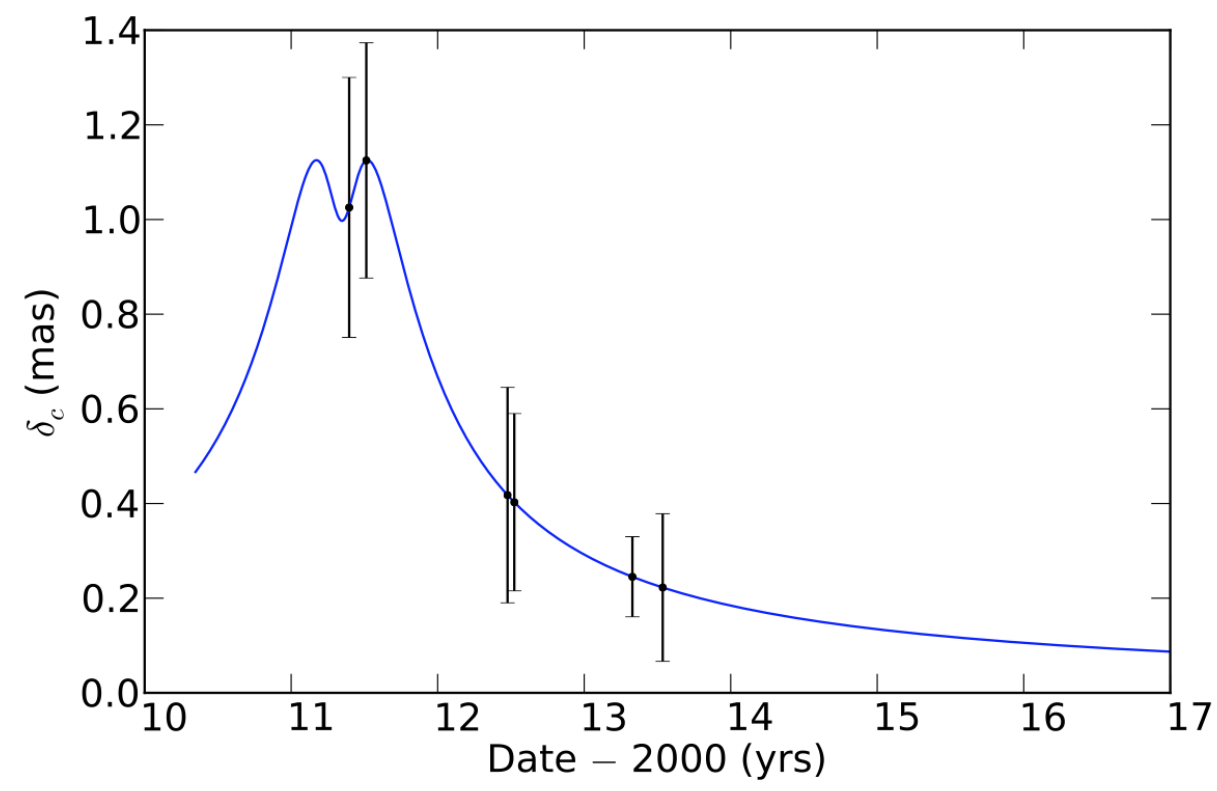

Figure 9. The astrometric deviation from linear proper motion when a $10 \mathrm{M}_{\odot}$ black hole at 4 kpc lenses a background Galactic bulge star at $8 \mathrm{kpc}$ is shown in blue. Our observational errors and time sampling for the candidate black hole lens, OB110022, are shown as black error bars. Poor seeing ( $>1$ ") resulted in particularly large error bars in 2011 and 2012 and yield only linear motions with large uncertainties, preventing us from constraining the lens mass at this time. However, additional measurements in 2015 and 2016 will show detectable deviations from linearity if the lens is a black hole $>5 \mathrm{M}_{\odot}$.

National Science Foundation (United States), the National Research Council (Canada), CONICYT (Chile), the Australian Research Council (Australia), Ministério da Ciência, Tecnologia e Inovação (Brazil) and Ministerio de Ciencia, Tecnología e Innovación Productiva (Argentina). Some of the results were based on observations made with the NASA/ESA Hubble Space Telescope, obtained at the Space Telescope Science Institute, which is operated by the Association of Universities for Research in Astronomy, Inc., under NASA contract NAS 5-26555. These observations are associated with program 10172, 11671, 11708, 12318, 12667, and 13044.

\section{REFERENCES}

[1] Anderson, J. and King, I. R., "PSFs, Photometry, and Astronomy for the ACS/WFC," tech. rep. (Feb. 2006).

[2] Anderson, J. and van der Marel, R. P., "New Limits on an Intermediate-Mass Black Hole in Omega Centauri. I. Hubble Space Telescope Photometry and Proper Motions," ApJ 710, 1032-1062 (Feb. 2010).

[3] Piotto, G., Milone, A. P., Anderson, J., Bedin, L. R., Bellini, A., Cassisi, S., Marino, A. F., Aparicio, A., and Nascimbeni, V., "Hubble Space Telescope Reveals Multiple Sub-giant Branch in Eight Globular Clusters," ApJ 760, 39 (Nov. 2012).

[4] van der Marel, R. P., Anderson, J., Bellini, A., Besla, G., Bianchini, P., Boylan-Kolchin, M., Chaname, J., Deason, A., Do, T., Guhathakurta, P., Kallivayalil, N., Lennon, D., Massari, D., Meyer, E., Platais, I., Sabbi, E., Sohn, S. T., Soto, M., Trenti, M., and Watkins, L., "Local Group and Star Cluster Dynamics from HSTPROMO: The Hubble Space Telescope Proper Motion Collaboration," in [Astronomical Society of the Pacific Conference Series], Seigar, M. S. and Treuthardt, P., eds., Astronomical Society of the Pacific Conference Series 480, 43 (Mar. 2014).

[5] Genzel, R., Schödel, R., Ott, T., Eisenhauer, F., Hofmann, R., Lehnert, M., Eckart, A., Alexander, T., Sternberg, A., Lenzen, R., Clénet, Y., Lacombe, F., Rouan, D., Renzini, A., and Tacconi-Garman, L. E., 
"The Stellar Cusp around the Supermassive Black Hole in the Galactic Center," ApJ 594, 812-832 (Sept. 2003).

[6] Ghez, A. M., Salim, S., Weinberg, N. N., Lu, J. R., Do, T., Dunn, J. K., Matthews, K., Morris, M. R., Yelda, S., Becklin, E. E., Kremenek, T., Milosavljevic, M., and Naiman, J., "Measuring Distance and Properties of the Milky Way's Central Supermassive Black Hole with Stellar Orbits," ApJ 689, 1044-1062 (Dec. 2008).

[7] Lu, J. R., Ghez, A. M., Hornstein, S. D., Morris, M. R., Becklin, E. E., and Matthews, K., "A Disk of Young Stars at the Galactic Center as Determined by Individual Stellar Orbits," ApJ 690, 1463-1487 (Jan. 2009).

[8] Gillessen, S., Eisenhauer, F., Trippe, S., Alexander, T., Genzel, R., Martins, F., and Ott, T., "Monitoring Stellar Orbits Around the Massive Black Hole in the Galactic Center," ApJ 692, 1075-1109 (Feb. 2009).

[9] Yelda, S., Ghez, A. M., Lu, J. R., Do, T., Meyer, L., Morris, M. R., and Matthews, K., "Properties of the Remnant Clockwise Disk of Young Stars in the Galactic Center," ApJ 783, 131 (Mar. 2014).

[10] Stolte, A., Ghez, A. M., Morris, M., Lu, J. R., Brandner, W., and Matthews, K., "The Proper Motion of the Arches Cluster with Keck Laser-Guide Star Adaptive Optics," ApJ 675, 1278-1292 (Mar. 2008).

[11] Clarkson, W. I., Ghez, A. M., Morris, M. R., Lu, J. R., Stolte, A., McCrady, N., Do, T., and Yelda, S., "Proper Motions of the Arches Cluster with Keck Laser Guide Star Adaptive Optics: The First Kinematic Mass Measurement of the Arches," ApJ 751, 132 (June 2012).

[12] Hußmann, B., Stolte, A., Brandner, W., Gennaro, M., and Liermann, A., "The present-day mass function of the Quintuplet cluster based on proper motion membership," AAP 540, A57 (Apr. 2012).

[13] Rochau, B., Brandner, W., Stolte, A., Gennaro, M., Gouliermis, D., Da Rio, N., Dzyurkevich, N., and Henning, T., "Internal Dynamics and Membership of the NGC 3603 Young Cluster from Microarcsecond Astrometry," ApJL 716, L90-L94 (June 2010).

[14] Kudryavtseva, N., Brandner, W., Gennaro, M., Rochau, B., Stolte, A., Andersen, M., Da Rio, N., Henning, T., Tognelli, E., Hogg, D., Clark, S., and Waters, R., "Instantaneous Starburst of the Massive Clusters Westerlund 1 and NGC 3603 YC," ApJL 750, L44 (May 2012).

[15] Hog, E., Novikov, I. D., and Polnarev, A. G., "MACHO photometry and astrometry." AAP 294, 287-294 (Feb. 1995).

[16] Miyamoto, M. and Yoshii, Y., "Astrometry for Determining the MACHO Mass and Trajectory," $A J$ 110, 1427 (Sept. 1995).

[17] Walker, M. A., "Microlensed Image Motions," ApJ 453, 37 (Nov. 1995).

[18] Jeong, Y., Han, C., and Park, S.-H., "The Effect of Bright Lenses on the Astrometric Observations of Gravitational Microlensing Events," ApJ 511, 569-573 (Feb. 1999).

[19] Bennett, D. P., Becker, A. C., Quinn, J. L., Tomaney, A. B., Alcock, C., Allsman, R. A., Alves, D. R., Axelrod, T. S., Calitz, J. J., Cook, K. H., Drake, A. J., Fragile, P. C., Freeman, K. C., Geha, M., Griest, K., Johnson, B. R., Keller, S. C., Laws, C., Lehner, M. J., Marshall, S. L., Minniti, D., Nelson, C. A., Peterson, B. A., Popowski, P., Pratt, M. R., Quinn, P. J., Rhie, S. H., Stubbs, C. W., Sutherland, W., Vandehei, T., Welch, D., MACHO Collaboration, and MPS Collaboration, "Gravitational Microlensing Events Due to Stellar-Mass Black Holes," ApJ 579, 639-659 (Nov. 2002).

[20] Gould, A. and Yee, J. C., "Microlens Masses from Astrometry and Parallax in Space-based Surveys: From Planets to Black Holes," ApJ 784, 64 (Mar. 2014).

[21] Wizinowich, P. L., Le Mignant, D., Bouchez, A. H., Campbell, R. D., Chin, J. C. Y., Contos, A. R., van Dam, M. A., Hartman, S. K., Johansson, E. M., Lafon, R. E., Lewis, H., Stomski, P. J., Summers, D. M., Brown, C. G., Danforth, P. M., Max, C. E., and Pennington, D. M., "The W. M. Keck Observatory Laser Guide Star Adaptive Optics System: Overview," PASP 118, 297-309 (Feb. 2006).

[22] Lu, J. R., Exploring the origins of the young stars in the central parsec of the Galaxy with stellar dynamics, $\mathrm{PhD}$ thesis, University of California, Los Angeles (2008).

[23] Lu, J. R., Ghez, A. M., Yelda, S., Do, T., Clarkson, W., McCrady, N., and Morris, M., "Recent results and perspectives for precision astrometry and photometry with adaptive optics," in [Society of Photo-Optical Instrumentation Engineers (SPIE) Conference Series], Society of Photo-Optical Instrumentation Engineers (SPIE) Conference Series $\mathbf{7 7 3 6}$ (July 2010). 
[24] Yelda, S., Lu, J. R., Ghez, A. M., Clarkson, W., Anderson, J., Do, T., and Matthews, K., "Improving Galactic Center Astrometry by Reducing the Effects of Geometric Distortion," ApJ 725, 331-352 (Dec. 2010).

[25] Fritz, T., Gillessen, S., Trippe, S., Ott, T., Bartko, H., Pfuhl, O., Dodds-Eden, K., Davies, R., Eisenhauer, F., and Genzel, R., "What is limiting near-infrared astrometry in the Galactic Centre?," MNRAS 401, 1177-1188 (Jan. 2010).

[26] Flicker, R., "PSF Reconstruction for Keck AO," (Oct. 2008).

[27] Jolissaint, L., Neyman, C., Christou, J., and Wizinowich, P., "Adaptive optics point spread function reconstruction project at W. M. Keck Observatory: first results with faint natural guide stars," in [Society of Photo-Optical Instrumentation Engineers (SPIE) Conference Series], Society of Photo-Optical Instrumentation Engineers (SPIE) Conference Series 8447 (July 2012).

[28] Rigaut, F., Neichel, B., Boccas, M., d'Orgeville, C., Vidal, F., van Dam, M. A., Arriagada, G., Fesquet, V., Galvez, R. L., Gausachs, G., Cavedoni, C., Ebbers, A. W., Karewicz, S., James, E., Lührs, J., Montes, V., Perez, G., Rambold, W. N., Rojas, R., Walker, S., Bec, M., Trancho, G., Sheehan, M., Irarrazaval, B., Boyer, C., Ellerbroek, B. L., Flicker, R., Gratadour, D., Garcia-Rissmann, A., and Daruich, F., "Gemini multiconjugate adaptive optics system review - I. Design, trade-offs and integration," MNRAS 437, 23612375 (Jan. 2014).

[29] Neichel, B., Rigaut, F., Vidal, F., van Dam, M. A., Garrel, V., Carrasco, E. R., Pessev, P., Winge, C., Boccas, M., d'Orgeville, C., Arriagada, G., Serio, A., Fesquet, V., Rambold, W. N., Lührs, J., Moreno, C., Gausachs, G., Galvez, R. L., Montes, V., Vucina, T. B., Marin, E., Urrutia, C., Lopez, A., Diggs, S. J., Marchant, C., Ebbers, A. W., Trujillo, C., Bec, M., Trancho, G., McGregor, P., Young, P. J., Colazo, F., and Edwards, M. L., "Gemini multiconjugate adaptive optics system review - II. Commissioning, operation and overall performance," MNRAS 440, 1002-1019 (May 2014).

[30] McGregor, P., Hart, J., Stevanovic, D., Bloxham, G., Jones, D., Van Harmelen, J., Griesbach, J., Dawson, M., Young, P., and Jarnyk, M. A., "Gemini South Adaptive Optics Imager (GSAOI)," in [Ground-based Instrumentation for Astronomy], Moorwood, A. F. M. and Iye, M., eds., Society of Photo-Optical Instrumentation Engineers (SPIE) Conference Series 5492, 1033-1044 (Sept. 2004).

[31] Carrasco, E. R., Edwards, M. L., McGregor, P. J., Winge, C., Young, P. J., Doolan, M. C., van Harmelen, J., Rigaut, F. J., Neichel, B., Trancho, G., Artigau, E., Pessev, P., Colazo, F., Tigner, J., Mauro, F., Lührs, J., and Rambold, W. N., "Results from the commissioning of the Gemini South Adaptive Optics Imager (GSAOI) at Gemini South Observatory," in [Society of Photo-Optical Instrumentation Engineers (SPIE) Conference Series], Society of Photo-Optical Instrumentation Engineers (SPIE) Conference Series $\mathbf{8 4 4 7}$ (July 2012).

[32] Bedin, L. R., Piotto, G., Anderson, J., Cassisi, S., King, I. R., Momany, Y., and Carraro, G., " $\omega$ Centauri: The Population Puzzle Goes Deeper," ApJL 605, L125-L128 (Apr. 2004).

[33] Gratton, R. G., Carretta, E., and Bragaglia, A., "Multiple populations in globular clusters. Lessons learned from the Milky Way globular clusters," A\&AR 20, 50 (Feb. 2012).

[34] Dotter, A., Chaboyer, B., Jevremović, D., Baron, E., Ferguson, J. W., Sarajedini, A., and Anderson, J., "The ACS Survey of Galactic Globular Clusters. II. Stellar Evolution Tracks, Isochrones, Luminosity Functions, and Synthetic Horizontal-Branch Models," AJ 134, 376-390 (July 2007).

[35] Roediger, J. C., Courteau, S., Graves, G., and Schiavon, R. P., "Constraining Stellar Population Models. I. Age, Metallicity and Abundance Pattern Compilation for Galactic Globular Clusters," ApJS 210, 10 (Jan. 2014).

[36] Barber, C., Courteau, S., Roediger, J. C., and Schiavon, R. P., "Validation of optimized population synthesis through mock spectra and Galactic globular clusters," MNRAS 440, 2953-2968 (June 2014).

[37] Reynolds, M. T. and Miller, J. M., "A Swift Survey of Accretion onto Stellar-mass Black Holes," ApJ 769, 16 (May 2013).

[38] Agol, E. and Kamionkowski, M., "X-rays from isolated black holes in the Milky Way," MNRAS 334, 553-562 (Aug. 2002). 